\title{
Análisis cienciométrico de la producción científica acerca de la investigación sobre la evaluación de la implementación del e-learning en el periodo 2000-2015
}

\author{
DIANA MARCELA CARDONA-ROMÁN* \\ JENNY MARCELA SÁNCHEZ-TORRES ${ }^{\text {** }}$ \\ Universidad Nacional de Colombia - Bogotá D.C., Colombia. \\ Recibido el 27-11-2015; primera evaluación el 14-07-2016; \\ segunda evaluación el 15-03-2017; aceptado el 17-03-2017
}

\section{Resumen}

Se realiza una revisión documental para proporcionar un panorama de investigación sobre la evaluación de la implementación del e-learning a partir de la exploración en Scopus y Web of Science (WoS) desde el año 2000 a 2015. Se usa una metodología de análisis cienciométrico de cinco etapas: i) Recuperación, ii) Migración, iii) Análisis, iv) Visualización e v) Interpretación. Se analizó un conjunto de 1147 registros encontrándose que los países con mayor aporte fueron: Estados Unidos, Espańa, Reino Unido, Australia y Alemania. El análisis del panorama refleja una gama de temáticas relacionadas con el e-learning y diferentes áreas del saber, también, se encontró una escasa presencia de investigación y de autores de origen latinoamericano. Este trabajo permitirá que los investigadores identifiquen tendencias de los últimos quince años.

Palabras clave: educación virtual, evaluación, estudios bibliométricos, tendencias educativas.

\footnotetext{
Ingeniera de sistemas, magíster en Ingeniería de Sistemas y Computación, doctoranda en Ingeniería- Industria y Organizaciones de la Facultad de Ingeniería de la Universidad Nacional de Colombia sede Bogotá, Colombia. Contacto: dmcardonar@unal.edu.co

** Ingeniera de sistemas, magíster en Ingeniería de Sistemas y Computación, magíster en Gestión de la Innovación, doctora en Economía y Gestión de la Innovación. Profesora asociada de la Facultad de Ingeniería de la Universidad Nacional de Colombia sede Bogotá, Colombia. Contacto: jmsanchezt@unal.edu.co
} 
Scientometric analysis of the scientific research on the evaluation of the implementation of e-learning in the period 2000-2015

\section{Abstract}

A documental review to provide an overview about the evaluation of implementation of e-learning beginning with the exploration in Scopus and Web of Science (WoS) from 2000 to 2015 has been carried out. It was used a methodology for analyzing scientometric with five stages: i) Recovery, ii) Migration, iii) Analysis, iv) Display and v) Interpretation. A set of 1147 records were analyzed, and, it was found that countries with the greatest contribution to research in e-learning were: the United States, Spain, UK, Australia and Germany. The study shown a range of topics related to e-learning and knowledge in different areas, but also a lack of worldwide research and Latin American authors were identified. Finally, this work will allow researchers to identify trends over the last fifteen years.

Keywords: e-learning, evaluation, bibliometric studies, educational trends

Análise cientométrica da investigaçáo científica sobre a avaliação da implementaçáo do e-learning no período 2000-2015

\section{RESUMO}

Uma revisão da literatura para fornecer uma visão geral da pesquisa sobre a avaliação da execução do e-learning a partir da exploração no Scopus e Web of Science (WoS) desde o ano 2000 até 2015 foi feita. A metodologia usada para a análise cienciométrica tem as seguintes cinco fases: i) Recuperação, ii) Migraçóes, iii) Análise, iv) Visualização e v) Interpretação. Um conjunto de 1147 registros foram analisados, encontrou-se que os países com maior contribuição à pesquisa são: os Estados Unidos, a Espanha, o Reino Unido, a Austrália e a Alemanha. O trabalho mostrou uma variedade de tópicos relacionados com as diferentes áreas do conhecimento e ao e-learning, assim como, a falta de investigaçóes ao redor do mundo, e pesquisadores na América Latina. Finalmente, o trabalho permitirá aos pesquisadores identificar tendências presentadas ao longo dos últimos quinze anos.

Palavras-chave: educação virtual, avaliação, estudos bibliométricos, tendências educacionais 


\section{INTRODUCCIÓN}

Los cambios tecnológicos y sus avances a ritmos acelerados, generan tanto en las personas, como en las diferentes instituciones, respuestas adaptativas para mantenerse vigentes (García-Peñalvo \& Seoane-Pardo, 2015). En ese mismo sentido ha progresado el e-learning, tanto en su uso (Ghaeni \& Abdehagh, 2010), como en su estructura tecnológica, pedagógica y metodológica (Fernández-Rodríguez, Miralles \& Rainer, 2014; García-Peñalvo \& SeoanePardo, 2015).

Hablar de e-learning implica discutir de tecnología al servicio de la educación, pero es más que eso, de hecho no hay consenso frente a la definición, ni a su escritura (e-learning, eLearning, e-Learning, ELearning, y E-learning) como lo indican Piotrowski (2009) y Cardona-Román y SánchezTorres (2011), además de los múltiples sinónimos con que es denominado (Educación Virtual, Virtual Learning, e-training, Online Learning, Online Education, Web-Based Education, Web-Based Learning, Computer-Based Learning, Virtual Classrooms, Adult Education, Distance Learning, Distance Education, Collaborative Learning, Mixed Learning, Blended Learning, Mediated Learning, Digital Education, Cybereducation) (Bustamante García, 2013; Piotrowski, 2009; Schneckenberg, 2004). Con todo, e-learning es entendido como procesos de enseñanza-aprendizaje que se llevan a cabo a través de Internet, con una separación física entre el tutor y el estudiante (CardonaRomán \& Sánchez-Torres, 2011; García-Peñalvo, 2005; Rosenberg, 2001).

La implementación de e-learning en las instituciones educativas, no solamente requiere de ambientes de enseńanza/aprendizaje digital soportados en el desarrollo y uso de herramientas informáticas indicadas para este propósito (infraestructura tecnológica), sino que requiere de planeación, de un análisis considerable del contexto, de comunicación, de liderazgo, de apoyo de las directivas, de suministro de recursos, de inversión económica, de visión institucional, de una estructura organizacional adecuada, entre otros (Fernández-Rodríguez et al., 2014; Ghaeni \& Abdehagh, 2010; Schneckenberg, 2004; Sharpe, Benfield \& Francis, 2006).

Además de lo anterior, es de vital importancia realizar evaluaciones del sistema de e-learning, que contribuyan tanto a reconocer su valor, como su eficiencia. Igualmente para que la sociedad, en general, la acepte y comprenda, elementos clave para el desarrollo y la expansión del e-learning (Raspopovic, Jankulovic, Runic \& Lucic, 2014).

Sin embargo, las evaluaciones no siempre se realizan al final de un ciclo, sino que pueden ser llevadas a cabo en cualquier momento del ciclo programático 
(Naciones Unidas, 2009; Osuna \& Márquez, 2000). Esto es, que la evaluación del e-learning puede realizarse en cualquier etapa de su ciclo, bien sea en la planeación, en su implementación, en su ejecución o en su operación. Pues la evaluación del e-learning no es una actividad de una sola vez, todo lo contrario se trata de un complejo proceso paralelo sistemático para el desarrollo y la puesta en práctica o implementación del mismo (Raspopovic et al., 2014).

Para Naciones Unidas la evaluación es una valoración rigurosa e independiente de actividades finalizadas o en curso, para determinar en qué medida se están logrando los objetivos estipulados y en qué medida contribuyen a la toma de decisiones. La evaluación, al igual que el seguimiento, se puede aplicar a muchas cosas, incluidas una actividad, un proyecto, un programa, una estrategia, una política, un tema, un sector o una organización. La distinción clave entre las dos es que las evaluaciones son hechas de forma independiente para proporcionar a los gerentes y al personal una valoración objetiva sobre si están o no bien encaminados. Además, son más rigurosas en sus procedimientos, diseño y metodología, y generalmente implican un análisis más amplio. Sin embargo, los objetivos del seguimiento y la evaluación son muy similares: proporcionar información que ayude a tomar decisiones más acertadas, mejorar el desempeño y alcanzar los resultados planeados (Naciones Unidas, 2009).

En el ámbito de los estudios métricos, la cienciometría consiste en la medición y el análisis cuantitativo de la producción científica (en especial los artículos científicos), para investigar el desarrollo, estructura, dinámica, tendencias y relaciones de la práctica científica, entre innumerables oportunidades investigativas (Cruz-Ramírez, Escalona-Reyes, Cabrera-García \& Martínez-Cepena, 2015; Jiménez-Contreras, 2000; Michán \& Muńoz-Velasco, 2013). Se recurre a la cienciometría, toda vez que el proceso científico es susceptible de ser cuantificado a través de indicadores métricos de la información (Cruz-Ramírez et al., 2015) y la evaluación de la actividad científica-tecnológica se realiza para un periodo de tiempo determinado (Sotolongo, Guzman, García \& Sanz, 2008). Esto implica que el análisis de esta documentación escrita permitirá, pues, descubrir sus características y su evolución (Escorsa \& Maspons, 2001). Por lo anterior, el manejo de herramientas tecnológicas para la administración de la información es un elemento esencial para el desarrollo social y crecimiento económico (Katz \& Hilbert, 2003).

Algunas investigaciones han abordado el uso de la cienciometría para analizar la producción científica en e-learning (Baker \& Yacef, 2009; Maurer \& Khan, 2010) o la producción científica educativa de determinados contextos nacionales o regionales (Anta, 2008; Cruz-Ramírez et al., 2015; Diem \& 
Wolter, 2013; Hernández-Pozo \& Fernández Rius, 2013; Sánchez, 2012). Sin embargo, en los estudios de (Baker \& Yacef, 2009; Maurer \& Khan, 2010) el análisis que desarrollan acerca del e-learning es global, es decir, que abordan el análisis de los estudios publicados en e-learning de 2003 a 2008, mientras que este artículo se enfoca en el análisis cienciométrico sobre la evaluación de la implementación del e-learning en educación superior, esto es, un análisis especializado en artículos centrados en una rama del e-learning, con el objeto de generar el panorama en el tema de estudio propuesto.

En síntesis, el objetivo de este artículo es presentar el panorama en la evaluación de la implementación del e-learning en educación, como resultado del análisis cienciométrico de dos herramientas bibliográficas (Scopus y Web of Science, WoS), enmarcadas en el periodo de tiempo de 2000 a 2015. Y así dar respuesta a la pregunta general que motivó esta investigación ¿Cuál es el panorama académico relacionado con la evaluación de la implementación del e-learning en educación superior?

$\mathrm{Al}$ mismo tiempo, se buscó la respuesta a las preguntas de interés enumeradas a continuación:

1. ¿Cómo es la distribución de las publicaciones por año?

2. ¿Cuáles son los tipos de referencias encontrados en la revisión?

3. ¿De cuáles países son los autores que más publican en e-learning?

4. ¿Cuáles son las instituciones con mayor participación en la publicación de e-learning?

5. ¿Cuál es la relación entre los principales autores, las revistas y las editoriales?

6. ¿Cuáles son las redes de autores que más publicaciones realizan en e-learning y en qué áreas investigan?

7. ¿Qué otros temas o descriptores se relacionan con e-learning?

Los resultados de este análisis cienciométrico son presentados a partir de la siguiente estructura del artículo. En la sección 1 (introducción) se brindó una síntesis de la fundamentación teórica del e-learning, la evaluación del e-learning y la evaluación de la implementación de e-learning. En la sección 2 se describe la metodología y el diseño metodológico empleado. En la sección 3 de presentan los resultados obtenidos a partir del análisis realizado y en la sección 4 se exponen las conclusiones del trabajo. Por último se manifiestan los agradecimientos y se enumeran las referencias empleadas. 


\section{Metodología}

La metodología utilizada para la realización del análisis cienciométrico, es la manejada en 2013 por Michán y Muñoz-Velasco en una versión adaptada, la cual consiste en cinco etapas principales, conocidas como: recuperación, migración, análisis, visualización, interpretación (Michán \& Muñoz-Velasco, 2013). Los autores proponen todas las etapas de manera secuencial, la adaptación realizada para este trabajo, recae en que las últimas tres etapas se repiten para cada una de las consultas.

Tal y como se presenta en la Figura 1, la metodología abarca un conjunto básico de pasos que componen cada una de las principales etapas.

Figura 1. Metodología para la realización de un análisis cienciométrico.

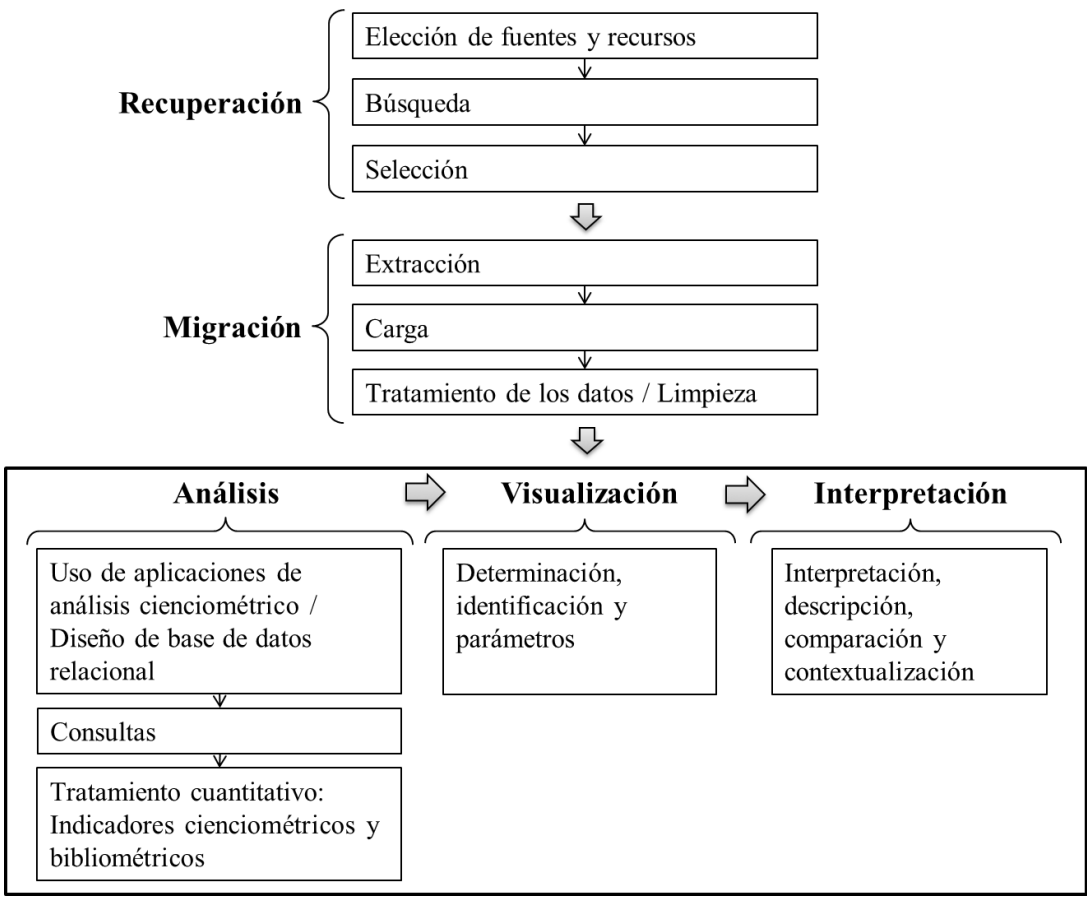

Fuente: Información adaptada de Michán \& Muñoz-Velasco (2013). 
A. Recuperación. Consiste en la selección de las fuentes de información, esto es la colección bibliográfica digital (base de datos) a utilizar. La búsqueda involucra el establecimiento de una consulta constituida por los términos, operadores y criterios adecuados para realizarla (Michán \& Muñoz-Velasco, 2013).

B. Migración. Comprende la extracción de metadatos de los registros seleccionados, la transferencia de la información extraída y la carga de esta en una nueva base de datos o una aplicación para el subsiguiente análisis. Posteriormente, los registros deben pasar por un proceso de curación para asegurarse que estén normalizados y depurados (Michán \& Muñoz-Velasco, 2013).

C. Análisis. Consiste en el procesamiento cuantitativo de la literatura. Uso o diseño de aplicaciones de software y la realización de consultas. En esta etapa comúnmente se aplica como procedimiento la obtención de indicadores bibliométricos (Michán \& Muñoz-Velasco, 2013). En el caso particular de este trabajo, se busca dar respuesta a cada una de las preguntas de interés propuestas.

D. Visualización. Se refiere a la obtención de figuras, gráficos, esquemas y mapas que reflejen las tendencias y los resultados de los análisis aplicados de una manera sintética, atractiva, estética, integral y amigable. La visualización generalmente se concentra en aquello que se considera que tiene una mayor contribución o que es más relevante, porque puede conducir a una mayor comprensión del tema, o que simplemente sea muy utilizado en el área de estudio (Michán \& Muñoz-Velasco, 2013).

E. Interpretación. Mediante la contextualización e interpretación de los resultados es posible establecer tendencias de investigación, representar influencias y comparaciones teóricas, metodológicas o sociales respecto a un grupo de investigación, institución, región, país, tema, disciplina o campo del conocimiento o modelo de estudio (Michán \& Muńoz-Velasco, 2013).

\section{Resultados}

Esta sección es presentada siguiendo cada una de las etapas establecidas en la metodología. Se expone en el mismo apartado los resultados del análisis, la visualización y la interpretación, porque estas etapas se realizan de manera recurrente para cada una de las consultas o preguntas de interés planteadas en la introducción. 


\section{A. Recuperación}

En el desarrollo de esta etapa se llevaron a cabo los siguientes tres pasos:

1. Selección de fuentes de información: Para este trabajo se seleccionaron las herramientas bibliográficas WoS publicada por ISI Thomson Reuters y Scopus publicada por la editorial Elsevier, toda vez que son bases de datos que se desempeñan como el referente para la medición de la producción científica (Cruz-Ramírez et al., 2015; HernándezPozo \& Fernández Rius, 2013), por el número de artículos publicados en reconocidas revistas académicas, por ser de las bases de datos bibliométricas más grandes, por estar actualizadas y por su impacto en la comunidad científica.

2. Búsqueda: Esta estuvo orientada por la pregunta principal ¿Cuál es el panorama académico relacionado con la evaluación de la implementación del e-learning en educación superior?, a partir de ella, se realiza la extracción de los términos usados para la construcción de la ecuación de búsqueda.

3. La exploración en las bases de datos estuvo orientada por la siguiente ecuación de búsqueda, en la cual se precisó una ventana tiempo de quince años desde el año 2000 hasta el 31 de marzo del año 2015:

4. "((measur* OR evaluat* OR asses*) AND (implement* OR implant*) AND ("e-learning" OR elearning OR "virtual learning" OR "virtual education" OR "online education" OR "web-based education" OR "web-based learning” OR "virtual program”) AND ("higher education" OR universit* OR HEI)) within 2000-2015".

5. Selección: Dado que la pregunta general se orientó en conocer el panorama de las publicaciones en torno a la evaluación de la implementación del e-learning, se seleccionó todas las referencias que aportó la búsqueda: WoS participó con 193 registros y Scopus contribuyó con 1112 registros.

\section{B. Migración}

Esta etapa tuvo el desarrollo de los siguientes tres pasos: 
1. Extracción: Se realizó la descarga del corpus de las búsquedas en WoS y Scopus, en dos archivos de texto en formato RIS, en total 1305 registros.

2. Carga: Se efectuó la carga del corpus (los archivos RIS) en la herramienta VantagePoint Versión 6.0.

3. Limpieza: Se procedió con la curación de los datos como: la eliminación de caracteres especiales, la unificación de los nombres (autor, publisher, keywords, source), la agrupación de los años de publicación y la eliminación de registros duplicados por la búsqueda en las dos bases de datos. Al finalizar el tratamiento de los datos, el corpus quedó con un total de 1147 registros.

\section{Análisis, visualización e interpretación}

Esta sección consistió en ejecutar las consultas relacionadas con las preguntas de interés planteadas en la introducción, e intentar dar respuesta al interrogante principal para conocer el panorama sobre la evaluación de la implementación del e-learning en educación superior. Tanto el análisis como la visualización se realizaron en diferentes herramientas de software como VantagePoint V6.0, Microsoft Excel profesional plus 2010, Fusión Tables de Google Drive y Target Map.

A continuación se presenta cada pregunta como la consulta (análisis), luego se realiza la visualización (tabla, mapa o gráfico) y posteriormente la interpretación de cada resultado.

\section{1. ¿Cómo es la distribución de las publicaciones por año?}

Se observa en la Figura 2 que las publicaciones por año tienen una tendencia de aumento, es decir, que el e-learning continúa siendo un área de investigación vigente, así como la evaluación del mismo. En ese sentido, se evidencia que los años con mayor número de referencias son los años 2013 con 154 y 2010 con 147 , es posible que esos picos puedan ser explicados por la periodicidad de las conferencias, toda vez que como se explica en la Tabla 1 , las referencias de este tipo son mayoría (46,6\%). Además que el lenguaje por excelencia en el mundo académico es el inglés con el $94 \%$ de las referencias, el 1,4\% de las referencias son en español y el porcentaje restante en otros idiomas. 
Figura 2. Distribución de las publicaciones por año sobre evaluación de la implementación de e-learning.

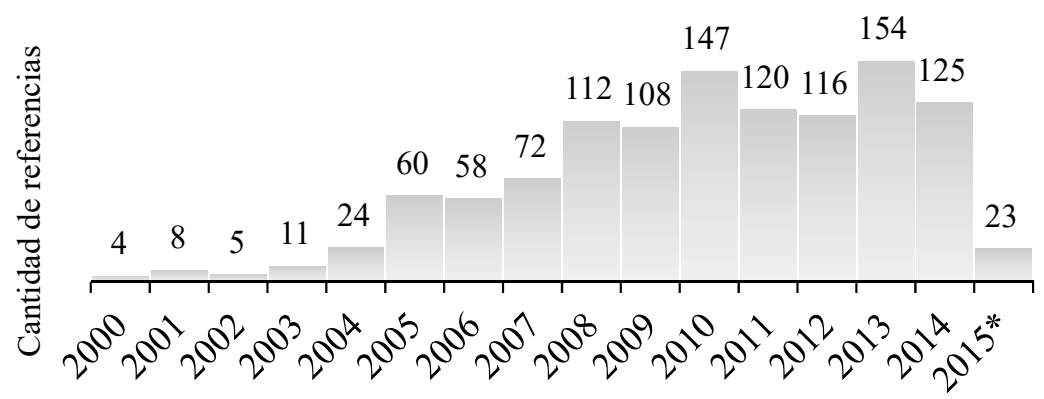

Años

Fuente: Elaboración propia en VantagePointV6 a partir del corpus de Scopus y WoS (2000 hasta 31/03/2015). * Nota: Para el ańo 2015 la consulta fue realizada hasta el día 31 del mes de marzo, por ello el número de publicaciones, en dicho año, es menor.

A partir de la producción académica registrada por autor y por año se identificaron las relaciones de trabajo de los principales autores y sus años de productividad, y se encontró que existe una fuerte relación entre los autores con mayor índice de publicación de artículos en e-learning y las publicaciones de los años 2010 a 2014, así como una menor proporción de publicaciones en los años 2000 a 2004.

\section{2. ¿Cuáles son los tipos de referencias encontrados en la revisión?}

La respuesta al interrogante se encuentra consignada en la Tabla 1, la cual indica la cantidad de artículos de acuerdo con el tipo de referencia que fue registrado. El tipo SD fue definido por las autoras, significa que hubo un total de 35 registros sin información en este campo, por lo tanto, fueron clasificadas bajo esta categoría.

Es notable en la Figura 3 que la mayoría $(46,6 \%)$ de las publicaciones encontradas corresponde a artículos de conferencias, es decir, que son documentos actualizados, aunque no necesariamente son evaluados con mayor rigurosidad. Estas publicaciones son seguidas por los artículos de revistas $(38,7 \%)$, que son documentos evaluados por pares, aunque no sean documentos tan actualizados, por los tiempos y procesos propios de revisión, edición y publicación de cada revista. 
Tabla 1. Referencias por tipo

\begin{tabular}{llcc}
\hline \multicolumn{1}{r}{ Tipo } & \multicolumn{1}{c}{ Descripción } & \# de referencias & Porcentaje \\
\hline CONF & Conferencia & 534 & $46,6 \%$ \\
JOUR & Journal / Revista & 444 & $38,7 \%$ \\
SER & Publicación serial & 73 & $6,4 \%$ \\
CHAP & Capítulo de libro & 46 & $4,0 \%$ \\
INPR & Publicación Impresa & 10 & $0,9 \%$ \\
BOOK & Libro & 5 & $0,4 \%$ \\
SD & Sin definir & 35 & $3,1 \%$ \\
\hline TOTAL & & 1147 & $100,0 \%$ \\
\hline
\end{tabular}

Fuente: Elaboración propia a partir del corpus de Scopus y WoS (2000 hasta 31/03/2015) previamente tratado en VantagePointV6.

Figura 3. Referencias por tipo sobre evaluación de la implementación de e-learning.

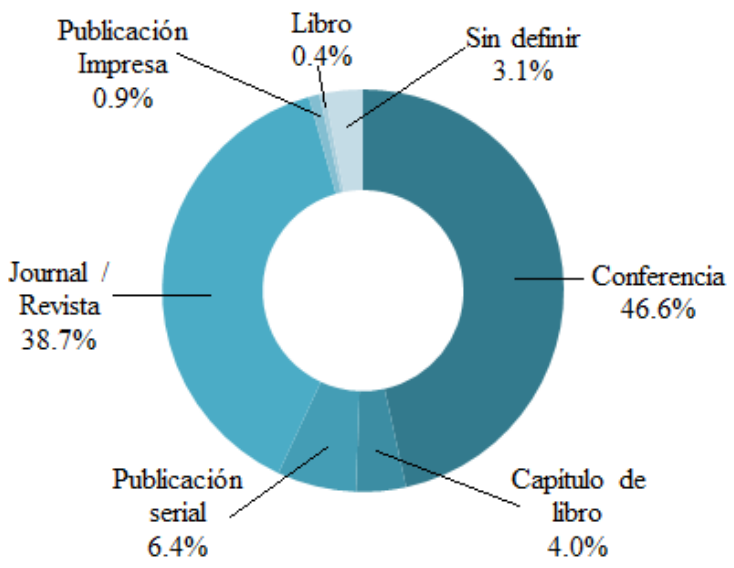

Fuente: Elaboración propia en Microsoft Excel a partir del corpus de Scopus y WoS (2000 hasta 31/03/2015) previamente tratado en VantagePointV6.

\section{3. ¿De qué países son los autores que más publican en e-learning?}

Al dar respuesta a la pregunta formulada, se identificó que este campo no estaba diligenciado en todos los registros del corpus; sin embargo, se obtuvo información del 96\% de las referencias. Se presenta en la Figura 4 los principales países de origen de los autores y se adicionan tres de los países americanos (Brasil, Colombia y México) con el objeto de realizar comparaciones de sus aportes al e-learning. 
Es Estados Unidos el país que mayor aporte hace con el 16,9\% de los autores con esta procedencia, seguido por España con el 7,8\%. Colombia presenta 12 autores, aunque solo es el $0,6 \%$ del aporte, es mayor que el realizado por México $(0,5 \%)$ y que el realizado por otros países del continente americano como Bolivia, Cuba, Chile, Ecuador, Nicaragua y Puerto Rico, cada uno con un autor procedente de estos países, los cuales aportan menos del $0,1 \%$ cada uno.

La visualización de la procedencia de las publicaciones se puede observar en la Figura 5, en la Figura 6 y en la Figura 7, las cuales detallan el origen de todos los autores que aportan al conocimiento en e-learning. Representar los hallazgos en el mapa (Figura 5) permite tener una mejor percepción de los aportes en e-learning, comprender que son los países desarrollados los que lideran las investigaciones en el tema de estudio, y evidenciar la concentración de aportes al conocimiento que tiene Europa (Figura 6) son componentes que favorecen la elaboración del panorama objeto de esta investigación.

Asimismo, visualizar la distribución porcentual de los países de origen de los autores (Figura 7) permite tener en cuenta la visión de los aportes que se realizan en diferentes países del mundo, donde un gran porcentaje $(23,4 \%)$ son realizados por una minoría de países.

Figura 4. Principales países de origen de los autores sobre evaluación de la implementación de e-learning.

Países de origen de los autores

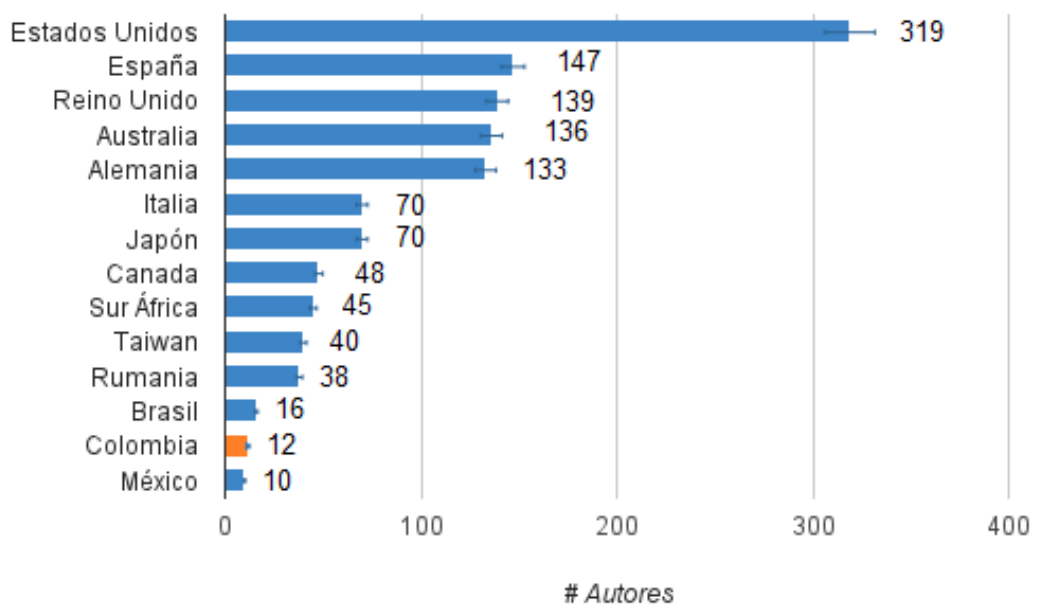

Fuente: Elaboración propia en Fusion Tables de Google a partir del corpus de Scopus y WoS (2000 hasta 31/03/2015). 
Figura 5. Mapa de procedencia de los autores en el tema de evaluación de la implementación de e-learning.

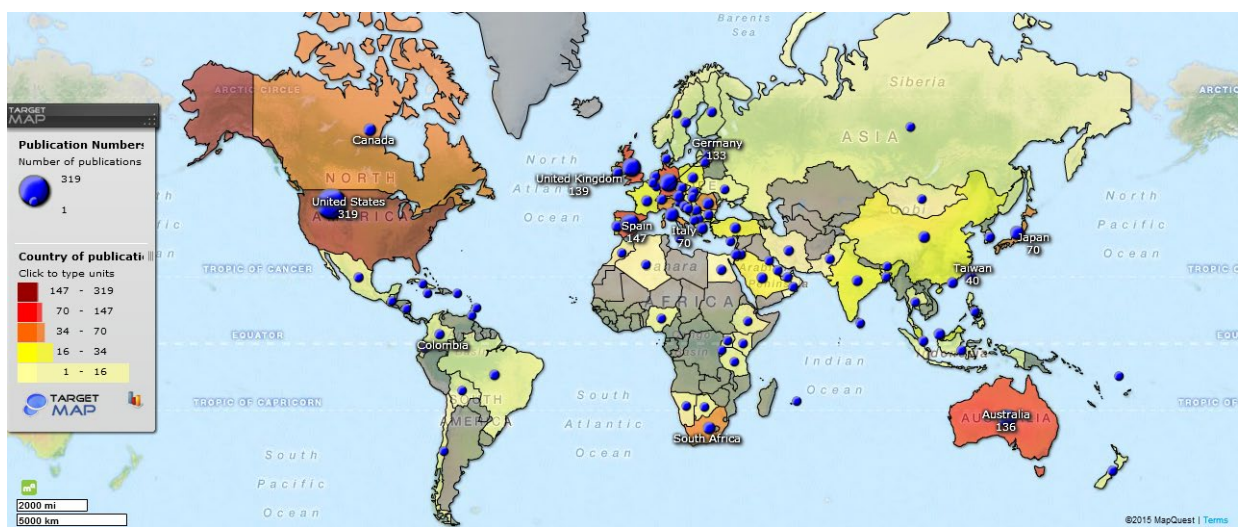

Fuente: Elaboración propia en TargetMap a partir del corpus de Scopus y WoS (2000 hasta 31/03/2015) y consulta realizada en Fusion Tables de Google, el enlace público de esta información es: http://www.targetmap.com/viewer.aspx?reportId=41589

Figura 6. Mapa europeo de procedencia de los autores en el tema de evaluación de la implementación de e-learning.

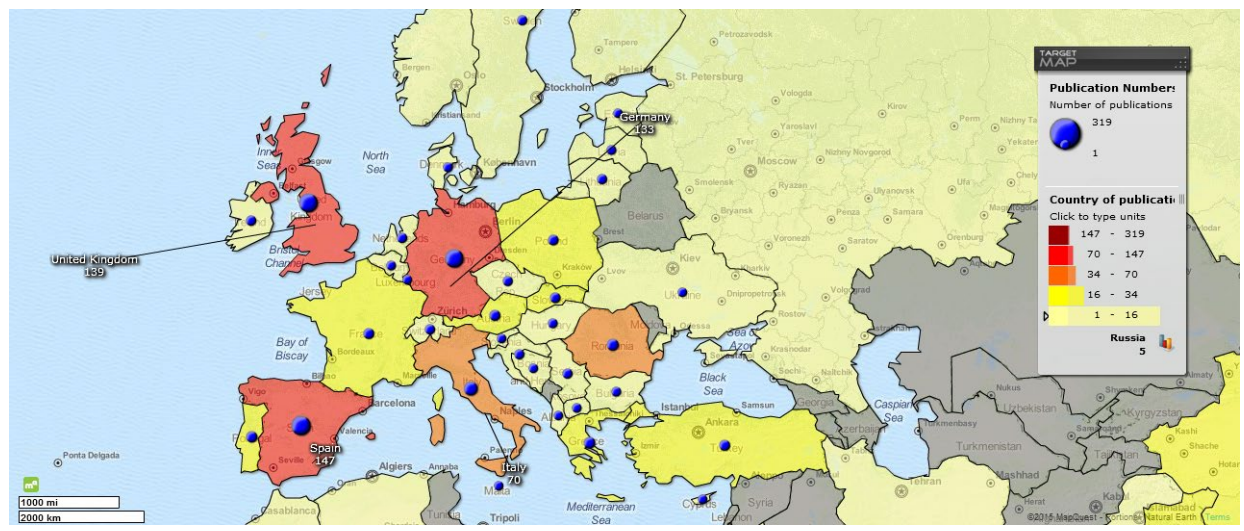

Fuente: Elaboración propia en TargetMap a partir del corpus de Scopus y WoS (2000 hasta 31/03/2015) previamente tratado en Fusion Tables de Google. 


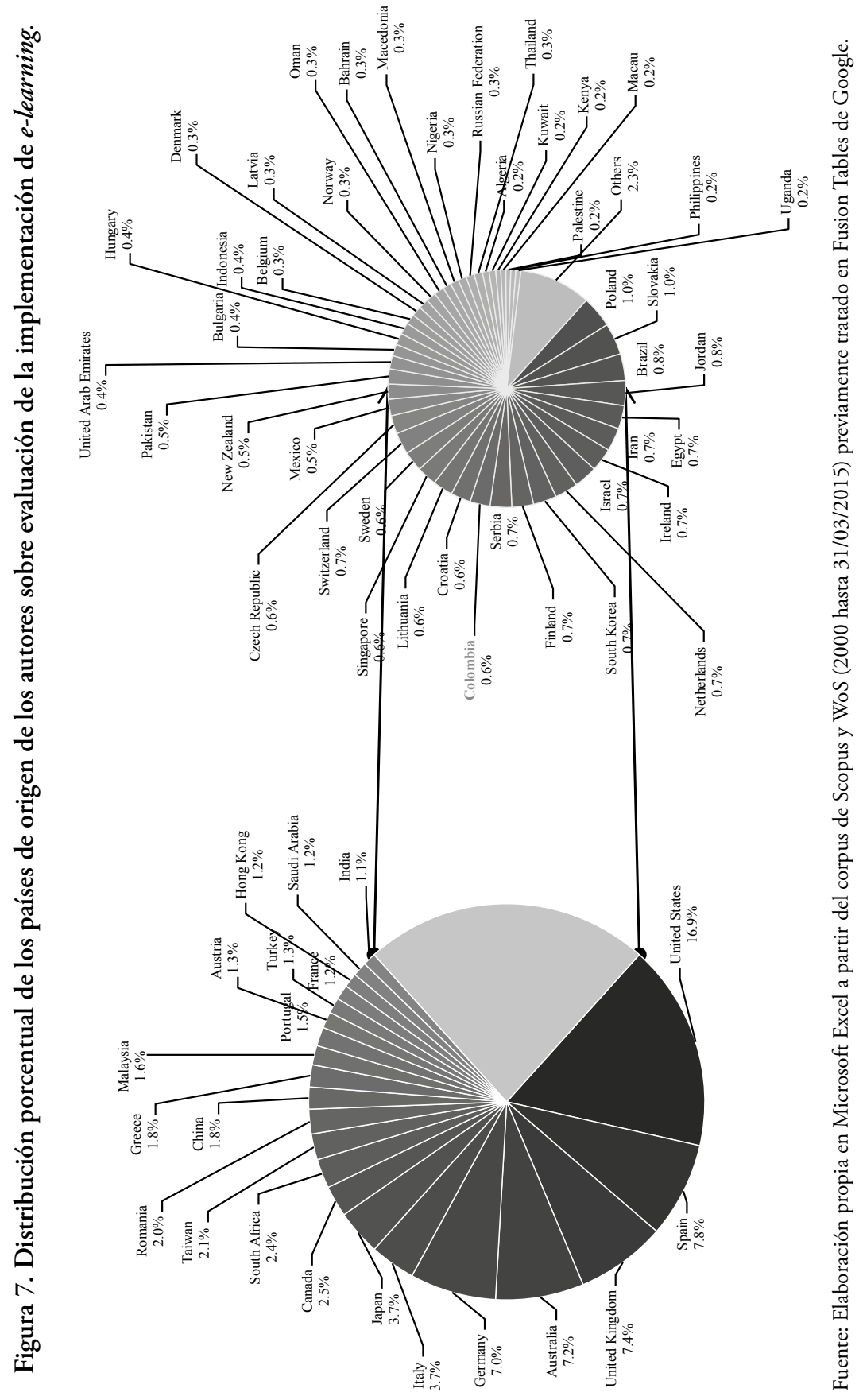




\section{4. ¿Cuáles son las instituciones con mayor participación en la publicación de e-learning?}

Existen diversas instituciones que participan en la publicación de temas relacionados con el e-learning. Convenientemente son las instituciones de educación superior (universidades) las que mayor aporte realizan a la generación de conocimiento en este campo.

La Figura 8 representa en el mapa la procedencia de las instituciones, y en la Figura 9 se observa, luego del análisis de las filiaciones de los autores, las 25 principales instituciones que aportan en e-learning. No sorprende que la UNED de España sea la institución que encabeza la producción académica, como quiera que ha sido una institución líder en el tema. Las universidades del estado de Arizona y South Australia, son las que preceden a la institución líder en cantidad de autores registrados con esta filiación.

Figura 8. Mapa de procedencia de las instituciones a las cuales están afiliados los autores que publican sobre la evaluación de la implementación de e-learning.

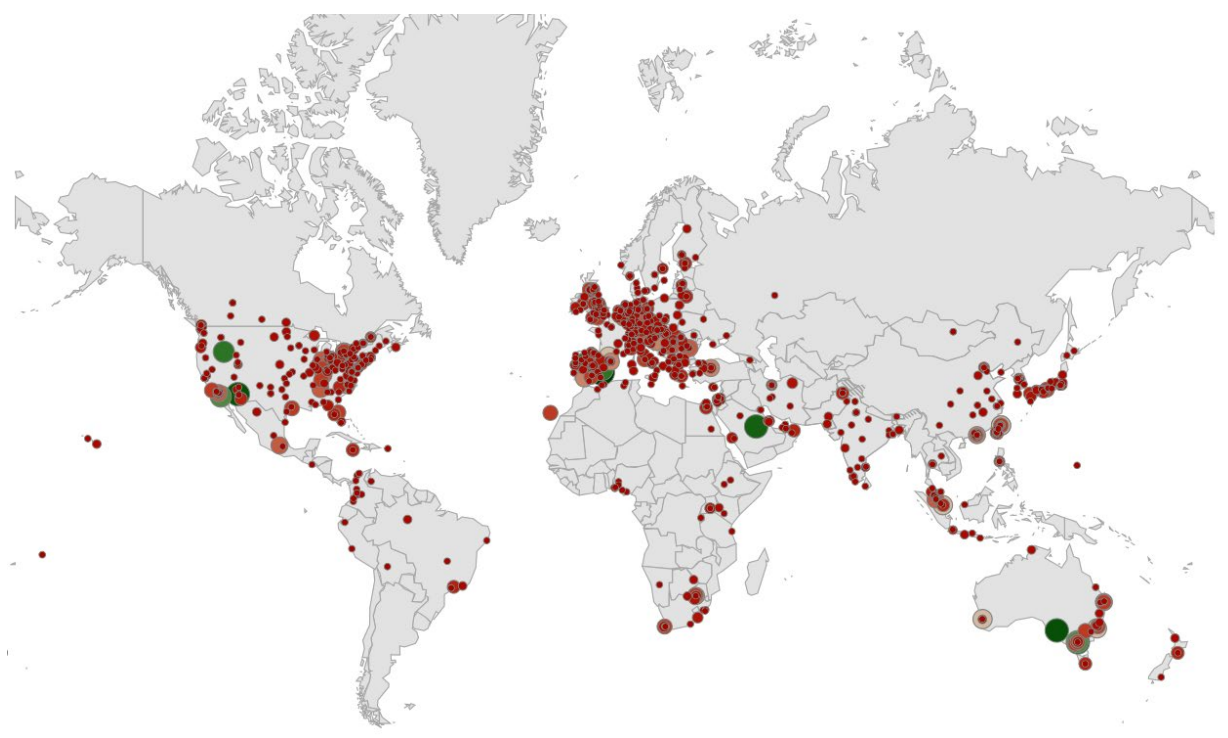

Fuente: Elaboración propia en Fusion Tables de Google a partir del corpus de Scopus y WoS (2000 hasta 31/03/2015). Nota: El tamańo de los círculos indica la cantidad de veces que apareció la institución y el color verde que hay mayor número, mientras que el color rojo indica que hay un menor número. 
Figura 9. Principales filiaciones de los autores.

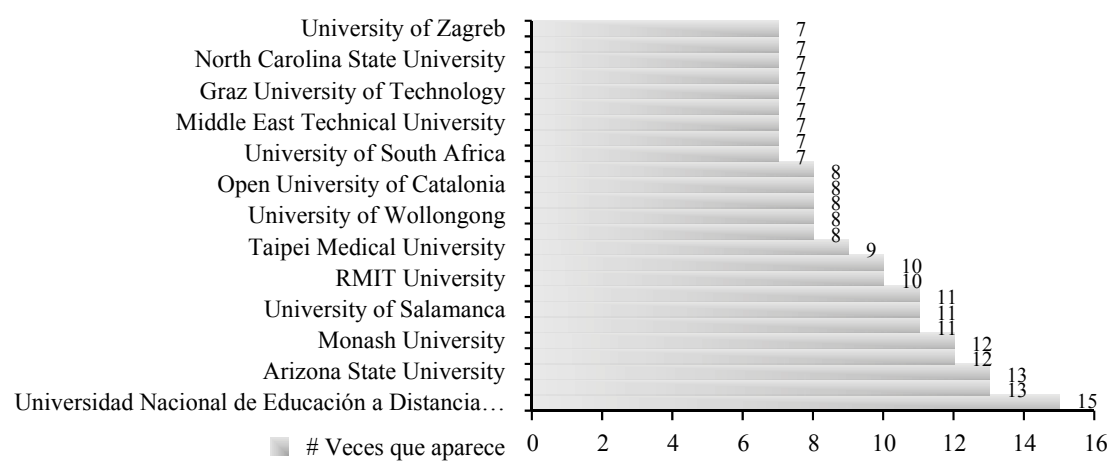

Fuente: Elaboración propia en Microsoft Excel a partir del corpus de Scopus y WoS (2000 hasta 31/03/2015) previamente tratado en Fusion Tables de Google.

\section{5. ¿Cuál es la relación entre los principales autores, las revistas y las editoriales?}

$\mathrm{Al}$ indagar por la relación existente entre los principales autores y las principales fuentes de publicación, se identificó en la Figura 10 las principales revistas (journals) donde los autores publican en el campo del e-learning, siendo la revista Computer \& Education, indexada en Colombia en la categoría más alta (A1), la que mayor cantidad de artículos presenta.

Figura 10. Principales fuentes de publicación.

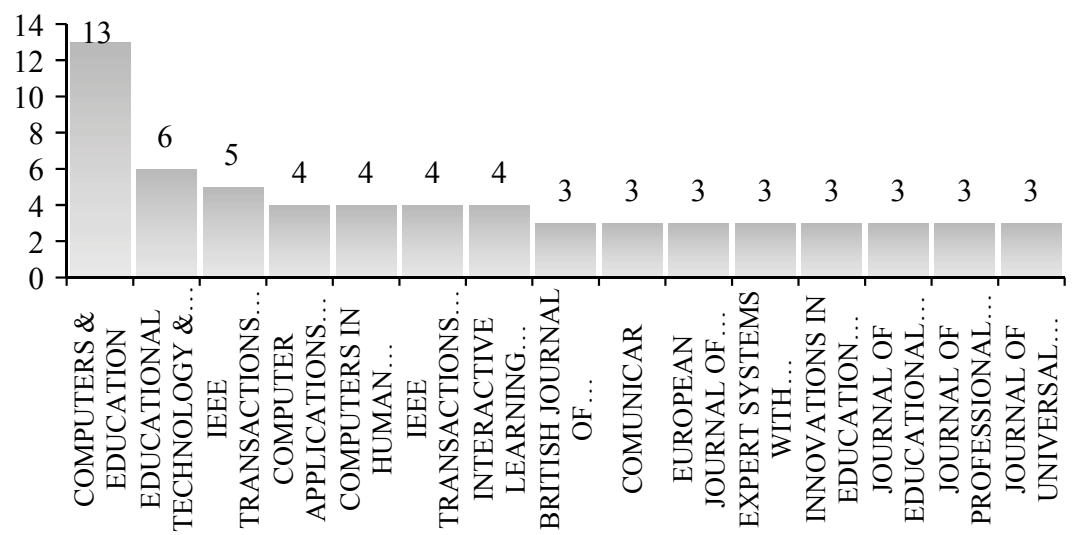


En la Figura 11 se muestra las relaciones de las principales revistas con los autores que mayor publicación efectúan, donde aparecen Educational Technology \& Society; IEEE Transactions on Education y Journal of Universal Computer Science.

Figura 11. Relaciones de los principales autores y las principales fuentes de publicación.

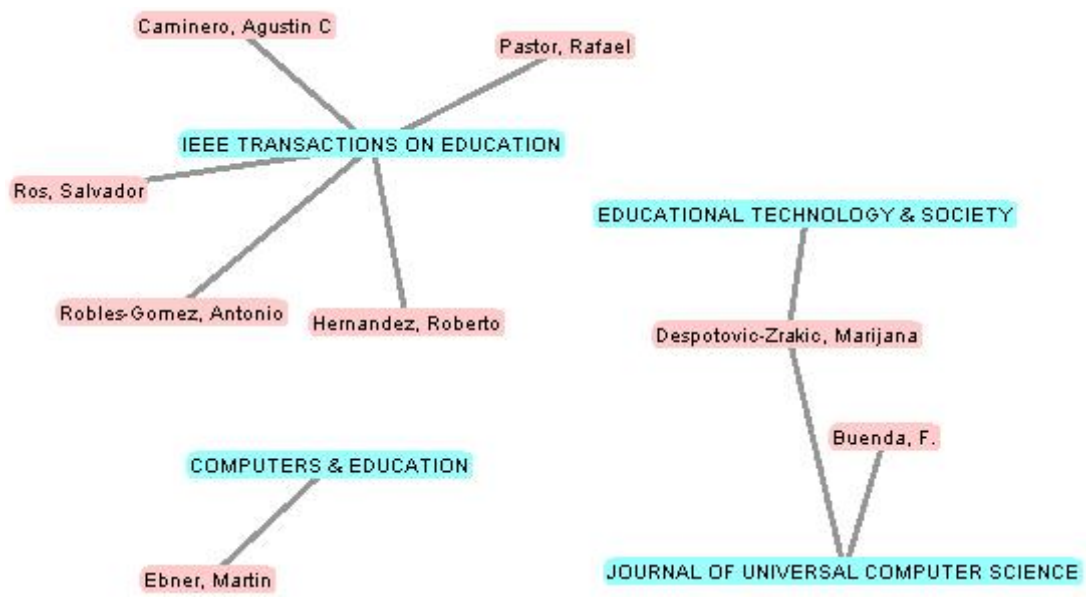

Fuente: Elaboración propia en VantagePointV6 a partir del corpus de Scopus y WoS (2000 hasta $31 / 03 / 2015)$.

Para conocer las principales editoriales que se relacionan con los autores es necesario consultar cuáles son esas editoriales que tienen mayor número de artículos asociadas a ellas. La respuesta se expone en la Figura 12 que contiene el listado de las veinte primeras editoriales, estando en el primer lugar la Academic Conference Limited con 67 registros, siendo este resultado coherente con la clasificación por tipo de referencia (mayor porcentaje en el tipo conferencia). Lo anterior explica que esta es una entidad que respalda la realización de conferencias en el ámbito del e-learning.

En la Figura 13 se presenta un extracto de las relaciones más importantes entre los principales autores y las principales casas editoriales, siendo la editorial Springer la que posee mejor relación con los autores que más documentos publicados conciernen en este estudio. Es indiscutible que los principales autores prefieran publicar sus trabajos de investigación en revistas (journal) que en conferencias, dado que las primeras tienen una mayor rigurosidad en su protocolo de evaluación, mientras que las segundas pueden no ser tan rigurosas. De allí que sea la editorial Springer la que presente esta importante 
relación con los principales autores. No obstante, la editorial Academic Conference Limited aparece en el gráfico relacional con un autor principal, lo que respalda el hecho de ser una entidad referente para el patrocinio y organización de eventos académicos.

\section{Figura 12. Principales editoriales.}

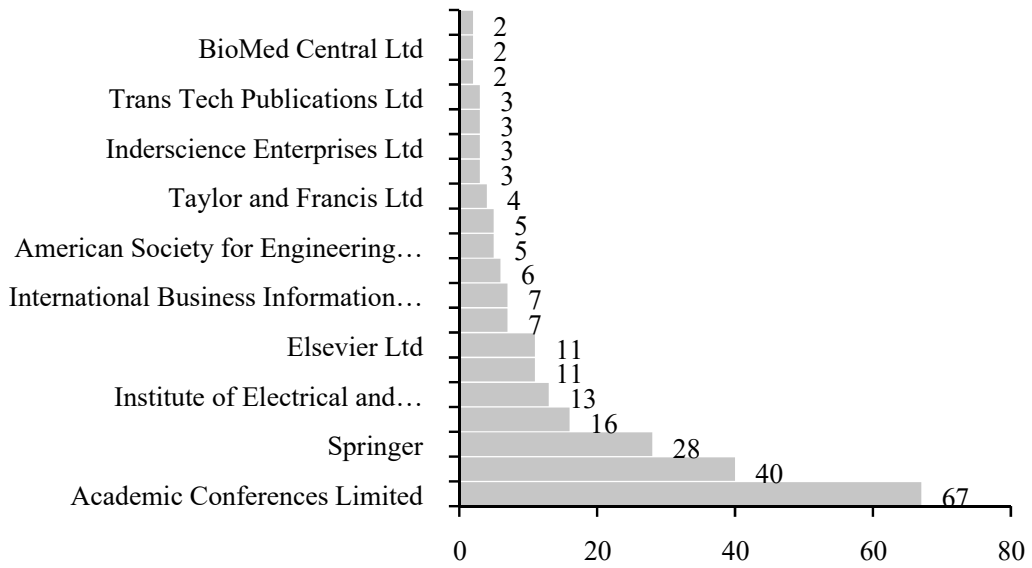

Fuente: Elaboración propia en VantagePointV6 a partir del corpus de Scopus y WoS (2000 hasta $31 / 03 / 2015)$.

Figura 13. Relaciones de los principales autores y las principales editoriales.

Academic Conferences Limited
American Society for Engineering Education

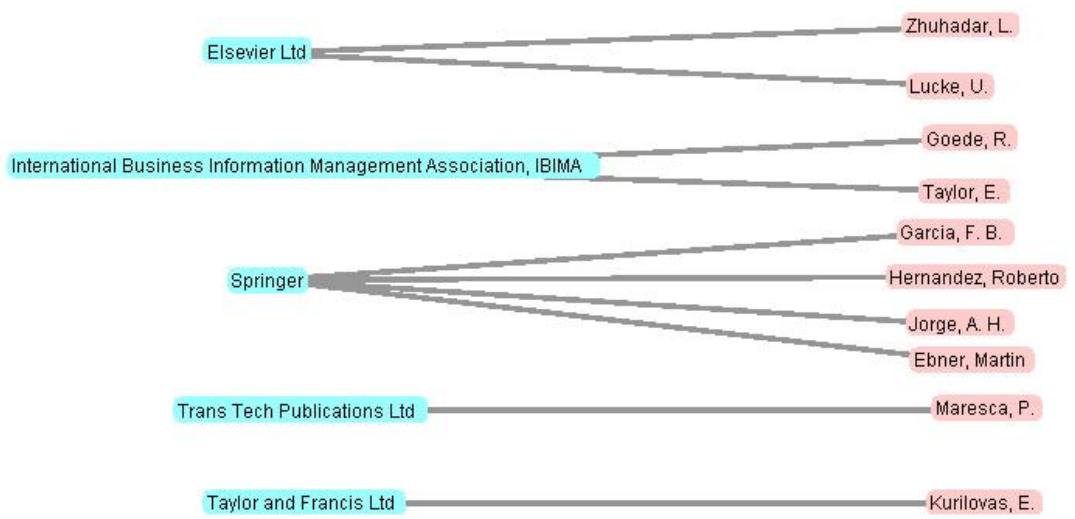

Fuente: Elaboración propia en VantagePointV6 a partir del corpus de Scopus y WoS (2000 hasta 31/03/2015). 


\section{6. ¿Cuáles son las redes de autores que más publicaciones realizan en e-learning y en qué áreas investigan?}

Para dar respuesta a la interrogante primero se consultó sobre los autores con mayor número de referencias en el corpus, los años de sus publicaciones y las citaciones que tenían, para ello se realizó la Tabla 2 que da cuenta de los principales autores y de sus referencias. Se considera sustancial mencionar que el campo de citaciones solamente tuvo datos en el 17\% del corpus, así que aunque su información no es significativa, puede ser orientadora en la prioridad de revisar los trabajos de los autores que tienen un mayor número de citaciones.

Se evidencia además que el autor con mayor número de citaciones registradas es Martín Ebner con 114 y fue a través de una publicación tipo journal del año 2007. Otro de los autores que no aparece en la tabla, pero que tiene 90 citaciones en su artículo tipo journal del año 2007 es Hassan Selim. No aparece referenciado habida cuenta que solo tiene una publicación, mientras que los autores que aparecen referenciados en la tabla cuentan con más de cuatro publicaciones.

Tabla 2. Autores con mayor número de publicaciones o referencias en el corpus

\begin{tabular}{|c|c|c|c|c|c|c|c|c|c|c|c|c|}
\hline \multirow[b]{2}{*}{ Autor } & \multirow{2}{*}{ 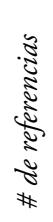 } & \multicolumn{10}{|c|}{ Años de las publicaciones/Referencias } & \multirow{2}{*}{ 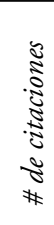 } \\
\hline & & $\begin{array}{l}\stackrel{2}{ } \\
\stackrel{2}{ }\end{array}$ & $\hat{\&}$ & $\underset{\sim}{\stackrel{ి}{ి}}$ & $\underset{\sim}{\stackrel{े}{~}}$ & 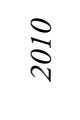 & $\underset{\text { ¿ }}{\bar{s}}$ & $\underset{\sim}{\stackrel{\sim}{~}}$ & $\underset{\sim}{n}$ & $\underset{\nu}{\stackrel{\varpi}{\Xi}}$ & $\stackrel{n}{\stackrel{n}{\delta}}$ & \\
\hline Castro, $M$ & 6 & & & $\sqrt{ }$ & $\sqrt{ }$ & $\sqrt{ } \sqrt{ }$ & $\sqrt{ }$ & & $\sqrt{ }$ & & & 0 \\
\hline García-Peñalvo, FJ. & 6 & & & & $\sqrt{ } \sqrt{ }$ & $\sqrt{ }$ & & $\sqrt{ }$ & $\sqrt{ }$ & $\sqrt{ }$ & & 0 \\
\hline Hernández, Roberto & 6 & & & & & $\sqrt{ } \sqrt{ }$ & $\sqrt{ }$ & $\sqrt{ }$ & $\sqrt{ }$ & $\sqrt{ }$ & & 3 \\
\hline Pastor, Rafael & 5 & & & & $\sqrt{ }$ & $\sqrt{ } \sqrt{ }$ & $\sqrt{ }$ & $\sqrt{ }$ & & & & 3 \\
\hline Ros, Salvador & 5 & & & & & $\sqrt{ } \sqrt{ }$ & $\sqrt{ }$ & & $\sqrt{ }$ & & & 3 \\
\hline Zhuhadar, $L$. & 5 & & & & $\sqrt{ }$ & $\sqrt{ } \sqrt{ }$ & & & & & $\sqrt{ }$ & 0 \\
\hline Ebner, Martín & 4 & & $\sqrt{ }$ & & & $\sqrt{ }$ & $\sqrt{ }$ & & & $\sqrt{ }$ & & 114 \\
\hline Garcia, FB. & 4 & & $\sqrt{ }$ & $\sqrt{ } \sqrt{ }$ & & & & & & $\sqrt{ }$ & & 0 \\
\hline González, Maria & 4 & $\sqrt{ }$ & & & $\sqrt{ } \sqrt{ }$ & & $\sqrt{ }$ & & & & & 5 \\
\hline Hege, Inga & 4 & & $\sqrt{ }$ & & $\sqrt{ } \sqrt{ }$ & & & & $\sqrt{ }$ & & & 20 \\
\hline Robles-Gómez, Antonio & 4 & & & & & $\sqrt{ }$ & $\sqrt{ }$ & $\sqrt{ }$ & $\sqrt{ }$ & & & 3 \\
\hline
\end{tabular}

Fuente: Elaboración propia a partir del corpus de Scopus y WoS (2000 hasta 31/03/2015) previamente tratado en VantagePointV6. 
En la Figura 14 se despliegan las redes entre los principales autores con los que realizan colaboración, en las cuales se identifican cinco grandes subredes con más de tres integrantes (encerrados en óvalo) y otras siete subredes de colaboración con solo dos integrantes (encerrados en rectángulos).

Para la elaboración de la Figura 14 se seleccionaron aquellos autores que tuvieran tres o más referencias en el corpus y se construyó la red con un mapa de autocorrelación. La línea más gruesa que une los grafos representa una correlación de trabajo entre dichos autores mayor a 0,75 (entre más cercano a 1 mayor es la correlación y colaboración entre los autores y más gruesa la línea). La línea continua delgada indica que la correlación entre los autores está en el rango de 0,5 a 0,75 , la línea discontinua es la correlación en el rango de 0,25 a 0,5 y la línea punteada indica que la correlación es muy baja o inexistente, siendo menor a 0,25 .

$\mathrm{Al}$ indagar sobre los temas que publican esas redes de autores, se encontró que la red que se encuentra en la parte superior (a) de la Figura 14 trabaja en temas de educación relacionados con el medio ambiente y su evaluación, en particular, con estudios oceánicos y sistemas climáticos. La red que se encuentra en la parte superior izquierda (b) se enfoca en temas de automatización de evaluaciones en aulas digitales, la obtención de competencias, tutores virtuales y la educación a distancia. La red que se encuentra en la parte inferior (c) investiga en educación enfocada el área médica (realidad virtual, enfoques educativos en medicina ocupacional y efectos de pacientes virtuales en cursos de pediatría). La red de autores ubicada al costado derecho (d) investiga sobre objetos de aprendizaje, su calidad y la evaluación de experiencias de aprendizaje en ambientes virtuales. La red de autores ubicada al costado izquierdo de la imagen (e) investiga en temas relacionados con el currículo y algoritmos computacionales para su predicción.

Lo anterior, indica que son diversas las áreas de investigación donde tiene cabida el e-learning, tanto en educación formal como no-formal, desde las ciencias de la educación, pasando por las ciencias económicas, administrativas y contables, las ciencias sociales y humanas, la ingeniería, arquitectura y urbanismo, la agronomía y veterinaria, las matemáticas y ciencias naturales; las ciencias de la salud, hasta las bellas artes. 
Figura 14. Redes de colaboración de autores sobre evaluación de la implementación de e-learning

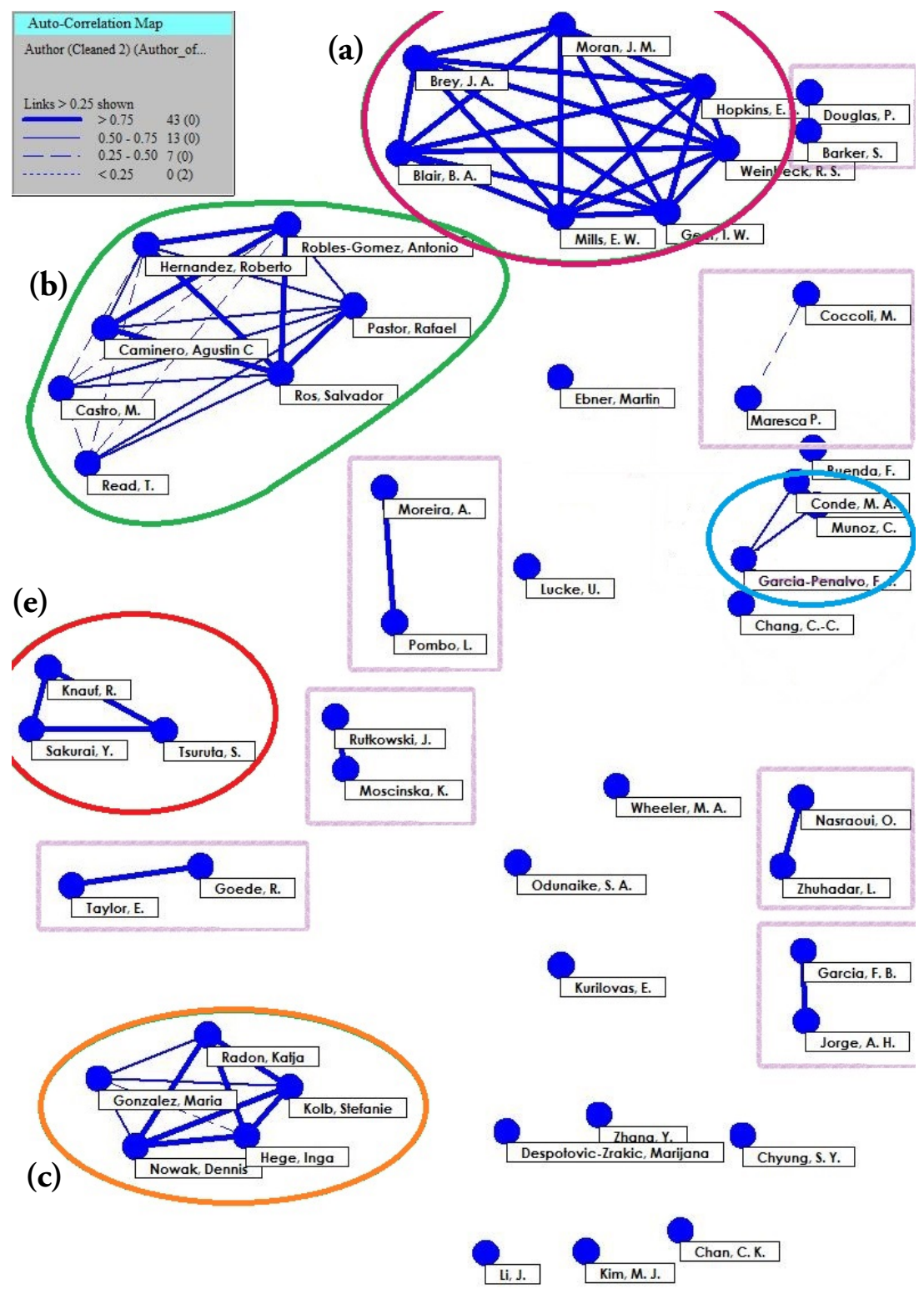

Fuente: Elaboración propia en VantagePointV6 a partir del corpus de Scopus y WoS (2000 hasta 31/03/2015). 


\section{7. ¿Qué otros temas o descriptores se relacionan con e-learning?}

Encontrar que otras palabras clave se relacionan con el término principal de la búsqueda que es e-learning es importante para determinar las relaciones existentes entre ellas, esto es, que son elementos que están involucrados y estrechamente relacionados.

En consonancia con lo anterior, en la Figura 15 se evidencia como los estudiantes (students) y la docencia (teaching) están ligados con el e-learning, además que estos se relacionan con el tema del currículo (curricula) y su diseño (design), siendo las palabras clave con mayor aparición. Esto significa que se está investigando en temas relacionados con los cursos, los contenidos, el diseño curricular y la relación con los principales actores (estudiantes y docentes).

Una subred (color naranja) que se evidencia muy relacionada entre ella, es la de artículos (article), humanos (buman), salud (health), instrucción asistida por computador (Computer-assisted instruction), educación (education), educación médica (medical education), calidad (quality) y estándar (estandar), lo que implica que el tema de e-learning está siendo ampliamente usado en temas relacionados con la educación en disciplinas médicas.

Otro tema de investigación procedente del análisis de la Figura 15 es el relacionado con la infraestructura tecnológica para la gestión del e-learning, donde los investigadores emplean diferentes palabras clave que aparecen dentro del grupo de las más utilizadas, aunque no necesariamente correlacionadas entre ellas, estas son: sistemas gestores de aprendizaje, Moodle, tecnologías de la información, interfaces de usuario, websites, sistemas de información, plataforma e-learning, ciencias de la computación, tecnología y software. Evidencian que al hablar de e-learning necesariamente se habla de tecnología y toda la investigación que ella derive.

Finalmente, nuestro interés es el de conocer como es la relación de la evaluación de la implementación del e-learning, en la Figura 15 se encontró un nodo de evaluación y otro de valoración, sin embargo, no se evidenció una fuerte relación de estos con el e-learning, aunque están presentes en el grafo relacional, puede indicar que se está trabajando en esos temas y aún queda bastante por investigar.

A su vez se indagó sobre el descriptor implementación, pero el término no se vio reflejado en el grafo. Es por ello que se realizó una consulta adicional con los sintagmas equivalentes para "implementación", allí se obtuvo un total de 17 palabras clave homólogas, como se muestra en la Figura 16, la cual también presenta cuatro sintagmas que no tienen una relación con el término principal del estudio (e-learning), pero que aparecen en las investigaciones referenciadas en el corpus analizado. 
Figura 15. Relación entre los principales descriptores de los artículos.

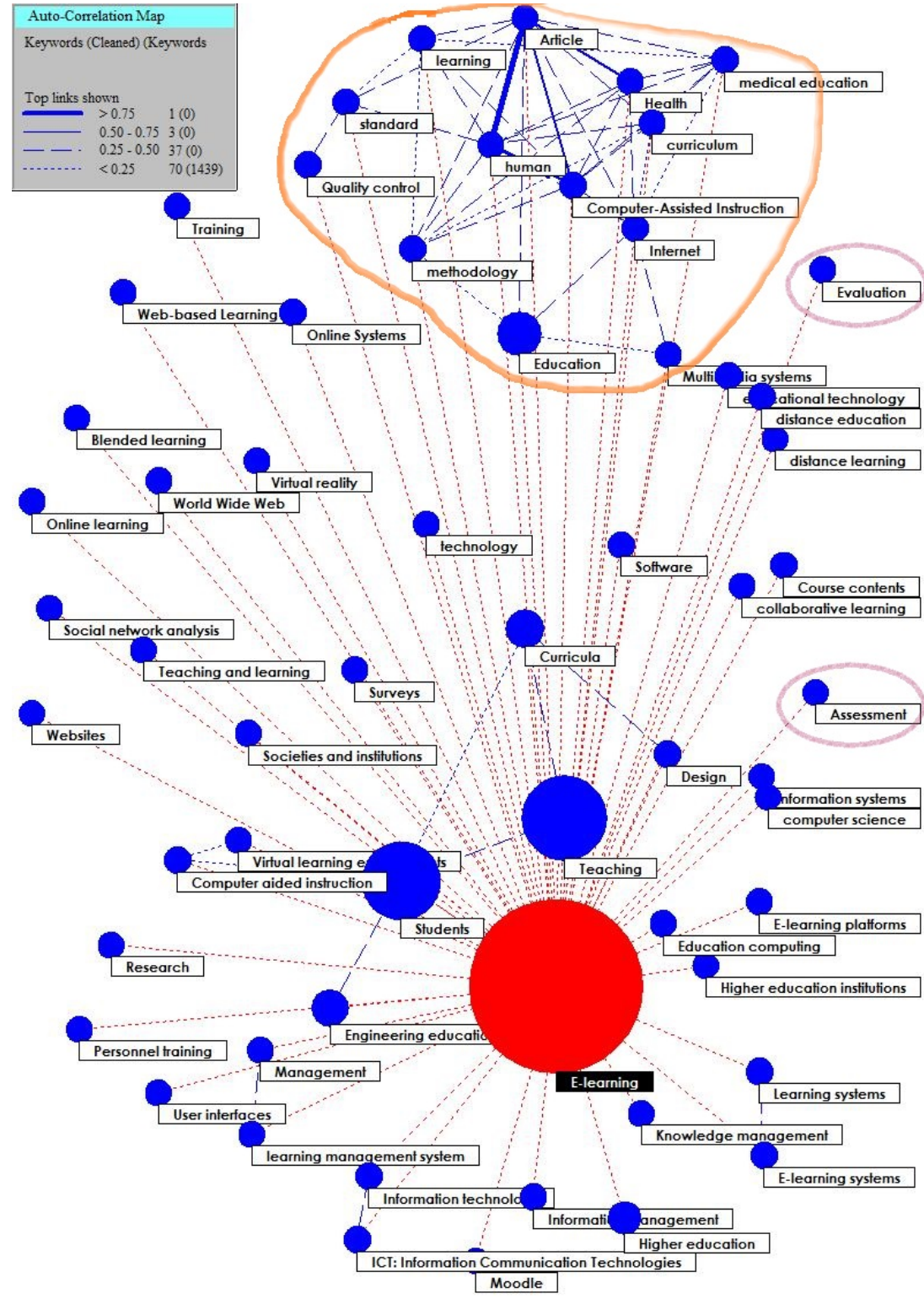

Fuente: Elaboración propia en VantagePointV6 a partir del corpus de Scopus y WoS (2000 hasta $31 / 03 / 2015)$. 
Lo anterior implica que aunque se tenga evidencia de investigaciones en la evaluación del e-learning, aún son pocos los estudios que involucran la evaluación en la implementación del mismo, por lo que es una oportunidad de investigación y de mover un poco la frontera del conocimiento en este campo. Es decir que la Figura 16 permite afirmar que se tiene pleno convencimiento que la investigación en temas que relacionen la implementación del e-learning y su evaluación es aún incipiente, lo que es demostrado por la escasez de apariciones de este descriptor clave en este estudio.

Figura 16. Relación entre los descriptores e-learning e implementación.

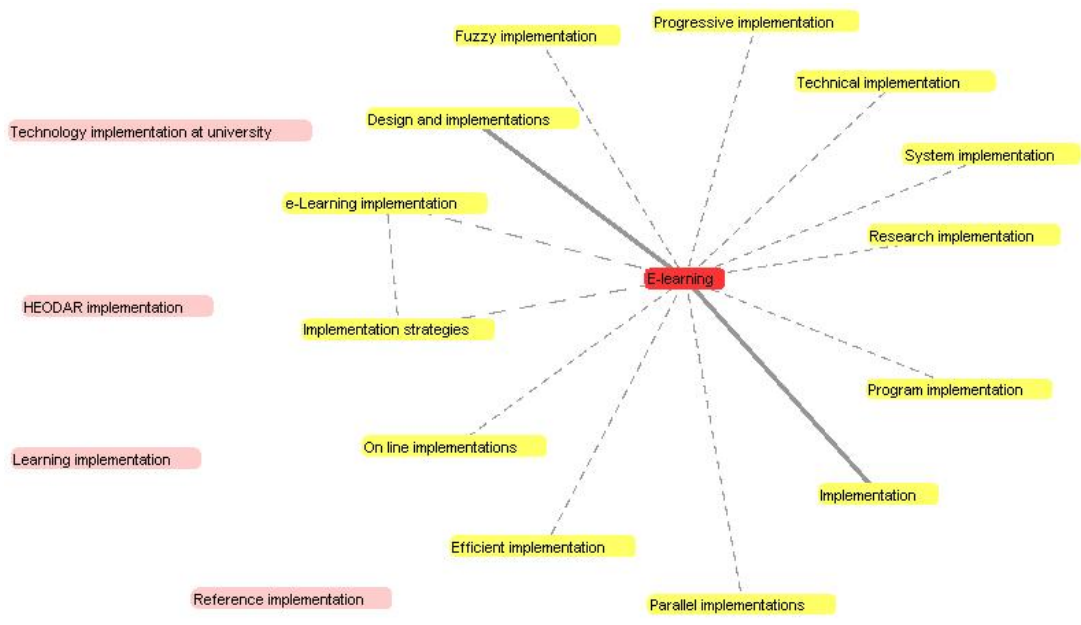

Fuente: Elaboración propia en VantagePointV6 a partir del corpus de Scopus y WoS (2000 hasta $31 / 03 / 2015)$.

\section{Conclusiones}

En este trabajo se sintetizó el panorama de la evaluación de la implementación del e-learning en el mundo, a través del análisis cienciométrico de la extracción de información de dos bases de datos (Scopus y WoS), como elemento diferenciador, se indicó las principales redes de colaboración de autores, las temáticas en las cuales realizaron sus aportes, las áreas o tópicos que se relacionan con la investigación del e-learning y la distribución geográfica de los autores.

Se procedió a dar respuesta a cada una de las preguntas de interés planteadas en la introducción, como se evidenció en la sección de resultados, 
cumpliendo así con el propósito general de la investigación, pues se conoció el panorama de la investigación alrededor de la evaluación de la implementación del e-learning en educación superior. En torno de ello, se encontró que las múltiples formas de escribir el vocablo (e-learning) y sus diversos sinónimos hacen que el análisis textual para descubrir nuevas relaciones entre autores, temáticas y áreas, sea más complejo.

Habida cuenta que se siguió un método sistemático de búsqueda y análisis de la información, el haber utilizado una ecuación de búsqueda, compuesta por términos y operadores lógicos, restringió el alcance de la exploración; por lo tanto, pudo haberse dejado de lado otros conceptos relacionados con la investigación y haber excluido artículos pertinentes.

Como se identificó en los resultados del artículo, las grandes áreas que emergen del análisis cienciométrico alrededor del e-learning y la educación son: la medicina, la salud, el medio ambiente, los estudios oceanográficos y marinos, la implementación de algoritmos computacionales para la predicción de currículo, el diseńo instruccional, los objetos de aprendizaje, la educación a distancia y la tecnología, son los más representativos.

Mediante el análisis de colaboración de los diversos autores en los documentos considerados en este trabajo, se realizaron algunas observaciones interesantes acerca de la naturaleza de las redes de colaboración académica, que por lo general está centrada en temas de interés de la red.

Por otra parte, la importancia de las conferencias regionales y los intercambios académicos debe tenerse en cuenta para comprender la forma en que las colaboraciones mencionadas se construyen y mantienen.

Son las instituciones educativas las que mayor aporte realizan al área del e-learning, sobresaliendo la Universidad Nacional de Educación a Distancia (UNED) de España como la institución líder en investigaciones en este ámbito.

Otro de los resultados interesantes fue el de los países de procedencia de los autores, allí se identificó que los autores que aportan en la investigación sobre e-learning proceden de diversos países, es decir, que aparte de ser un tema en crecimiento, es de interés mundial, toda vez que se identificaron procedencias de 95 países. Sin dejar de lado que los países que mayor aporte realizan a la investigación en e-learning son: Estados Unidos, Espańa, Reino Unido, Australia y Alemania, en orden de sus aportes, según la información disponible para este estudio.

Sumado a lo anterior, se identificó que la investigación en América Latina sobre la "evaluación de la implementación del e-learning" es un problema abordado de manera aún incipiente. 
Pese a haber dado respuesta a los interrogantes de interés, este estudio presentó algunas limitaciones, entre ellas, es que pese a haber utilizado los términos "implementación” y "evaluación” en la ecuación de búsqueda, fueron escasos los resultados de las referencias que se obtuvieron al combinar los dos términos, cerca del 3\%. Puesto que, el término "evaluación" tuvo una relación muy débil con e-learning y el término "implementación" no aparece como las palabras clave de mayor uso, esto genera una oportunidad para realizar investigaciones en este campo e implica realizar una revisión más detallada sobre la misma.

Por último, otra de las limitaciones del trabajo, fue conocer los aportes en cuanto a métodos, modelos o metodologías que evalúan la implementación de e-learning en educación superior. Por lo tanto, un trabajo interesante en esta línea sería ir a un nivel más profundo y analizar las contribuciones de los artículos que abordan la evaluación de la implementación.

\section{REFERENCIAS BIBLIOGRÁFICAS}

Anta, C. (2008). Análisis bibliométrico de la investigación educativa divulgada en publicaciones periódicas españolas entre 1990-2002. Revista Electronica de Investigacion Educativa, 10(1), 1-17. Recuperado de http://redie.uabc.mx/ index.php/redie/article/download/185/323

Baker, R. S. J. D. \& Yacef, K. (2009). The State of Educational Data Mining in 2009 : A Review and Future Visions. Journal of Educational Data Mining, 1(1), 3-16.

Bustamante García, A. F. (2013). Metodología para la evaluación del e-learning en educación superior presencial. SINAB - Universidad Nacional de Colombia. Universidad Nacional de Colombia. Recuperado de http:// www.bdigital.unal.edu.co/10774/

Cardona-Román, D. M. \& Sánchez-Torres, J. M. (2011). La educación a distancia y el e-learning en la Sociedad de la Información: una revisión conceptual. Revista UIS Ingenierías, 10(1), 29-52. Recuperado de http://revistas.uis. edu.co/index.php/revistauisingenierias/article/view/39-52

Cruz-Ramírez, M., Escalona-Reyes, M., Cabrera-García, S. \& Martínez-Cepena, M. (2015). Análisis Cienciométrico de las Publicaciones Educacionales Cubanas en la WoS y Scopus (2003-2012). Revista Española de Documentación Cientifica, 37(3), e058 (17). https://doi.org/10.3989/ redc.2014.3.1119

Diem, A. \& Wolter, S. C. (2013). The Use of Bibliometrics to Measure Research Performance in Education Sciences. Research in Higher Education, 54(1), 86-114. https://doi.org/10.1007/s11162-012-9264-5 
Escorsa, P. \& Maspons, R. (2001). La vigilancia tecnológica un requisito indispensable para la innovación. Recuperado de http://www.uned.es/expertouniversitario-gestion-I-D/bibliografia/bibliografia 2/BIBLIOGRAFIA/La vigilancia tecnologica un requisito indispensable para la innovacion.pdf

Fernández-Rodríguez, J. C., Miralles, F. \& Rainer, J. J. (2014). eLearning, TIC and the new teaching. Pensee Journal, 76(12), 51-56.

García-Peñalvo, F. J. (2005). Estado actual de los sistemas e-learning. Teoría de la Educación. Educación y Cultura en la Sociedad de la Información, 6(2), 1-5. Recuperado de http://www3.usal.es/ - teoriaeducacion/rev_numero_06_2/ n6_02_art_garcia_penalvo.htm

García-Peñalvo, F. J. \& Seoane-Pardo, A. M. (2015). Una revisión actualizada del concepto de eLearning. Décimo Aniversario. EKS, 16(1), 119-144.

Ghaeni, E. \& Abdehagh, B. (2010). A Model for Implementing E-Learning in Iranian Organizations. Recuperado de http://asianvu.com/bookstoread/etp/

Hernández-Pozo, M. D. R. \& Fernández Rius, L. E. (2013). Scientometric analysis of research from a feminist perspective. Acta Colombiana de Psicología, 16(2), 31-46. https://doi.org/10.14718/ACP.2013.16.2.3

Jiménez-Contreras, E. (2000). Los métodos bibliométricos Estado de la cuestión y aplicaciones. Science, 1 (Prmer Congreso Universitario de Ciencias de la Documentacio), 757-771. Recuperado de http://www.ucm.es/info/multidoc/multidoc/revista/num10/paginas/pdfs/ejcontreras.pdf

Katz, J. \& Hilbert, M. (2003). Los caminos hacia una sociedad de la información en América Latina y el Caribe. Santiago de Chile: Naciones Unidas.

Maurer, H. \& Khan, M. S. (2010). Research trends in the field of e-learning from 2003 to 2008: A scientometric and content analysis for selected journals and conferences using visualization. Interactive Technology and Smart Education, 7(1), 5-18. Recuperado de http://emeraldinsight.com. ezproxy.unal.edu.co/doi/pdfplus/10.1108/17415651011031617. https:// doi.org/10.1108/17415651011031617

Michán, L. \& Muñoz-Velasco, I. (2013). Scientometrics for the medical sciences: Definitions, applications and perspectives. Revista de Investigación En Educación Médica, 2(6), 100-106. Recuperado de http://riem.facmed. unam.mx/node/73. https://doi.org/10.1016/S2007-5057(13)72694-2

Naciones Unidas. (2009). Manual de Planificación, Seguimiento y Evaluación de los Resultados de Desarrollo. Nueva York, NY: PNUD.

Osuna, J. L. \& Márquez, C. (coords.) (2000). Guia para la evaluación de politicas públicas. Sevilla, España: Instituto de Desarrollo Regional, Fundación Universitaria. 
Piotrowski, M. (2009). Document-Oriented E-Learning Components. Magdeburg: Otto-Von-Guericke-Universitat. Recuperado de http://edoc.bibliothek.unihalle.de/servlets/DocumentServlet?id=7205

Raspopovic, M., Jankulovic, A., Runic, J. \& Lucic, V. (2014). Success Factors for e-Learning in a Developing Country: A Case Study of Serbia. International Review of Research in Open and Distance Learning, 15(3), 1-23. Recuperado de http://eric.ed.gov/?q=e-learning+review\&ft=on\&pg=2\&id=EJ1033100. https://doi.org/10.19173/irrodl.v15i3.1586

Rosenberg, M. J. (2001). E-learning: strategies for delivering knowledge in the digital age. Nueva York, NY: McGraw-Hill.

Sánchez, S. (2012). Análisis bibliométrico de los trabajos de investigación de la carrera de educación integral de la Universidad Nacional Abierta, Centro Local Carabobo. Unavisión: Revista del Centro Local Carabobo, 1(1), 91-102. Recuperado de http://biblo.una.edu.ve/ojs/index.php/UNAV/ article/view/1162

Schneckenberg, D. (2004). El e-learning transforma la educación superior. Educar, 23, 143-156. https://doi.org/10.5565/rev/educar.266

Sharpe, R., Benfield, G. \& Francis, R. (2006). Implementing a university e-learning strategy: levers for change within academic schools. Research in Learning Technology, 14(2), 135-151. https://doi.org/10.1080/ 09687760600668503

Sotolongo, G., Guzman, M. V., García, I. \& Sanz, E. (2008). Retos de la bibliometria: la vigilancia y Evaluacion de la Actividad Científico - Tecnológica. En Biblioteca Virtual de las Ciencias en Cuba (pp. 1-15). Cuba. Recuperado de http://www.bibliociencias.cu/gsdl/collect/eventos/index/assoc/HASH0 1a4/47cacf90.dir/doc.pdf 